\title{
On the Sensory Nerve Terminations in Clitoris in Human Adult.
}

\author{
By
}

\section{Katsuyoshi Yamada.} (山田癷 義)

(From the Anatomical Laboratory of Prof. H. Seto, Faculty of Medicine, Tohoku University, Sendai.)

(Received for publication, November 29, 1950)

After my previous studies ${ }^{11}$ ) on the innervation of clitoris in 10th month human embryo were done, I have entended to inquire into the same thesis in the adult continuously. Using 2 clitoris fixed in $10 \%$ neutral formol, I made them in $40 \mu$ serial transversal frozen sections and stained them with Seto's silver method. After having obtained very beautiful preparations, I have observed them in detail through the microscope and been able to supplement many of new knowledges.

\section{Histology of Clitoris in Adult.}

Previous to the description of the innervation of clitoris in adult, something shall be written on the fine structures of clitoris, because clitoris has been thought so long as an epitome of penis, though there are between both of them many histological differences, even in adult. Of course this fact has attracted the attention of some authorities, but there has been no description on it even in the authoritative text books. The histological differences between them are as follows.

1. Even in clitoris in adult exists corpus cavernosum urethra close beneath the corpora cavernosa clitoridis, like in the embryo. But it contains, unlike in the man, no urethra, becomes in its distal part small and narrow and pass finally into the corpus glandis clitoridis, however the passing part is indistinct sometimes.

2. The cavernous body of corpus glandis clitoridis is not surrounded by the strong connective capsule unlike in corpus glandis penis and communicates with the blood vessels especially with capillaries or small veins in the propria of the glans.

3. The common epithelium to the glans and the inner plate of præputium falls after the end stadium of embryonic life gradually in spliting from the apex glandis. In the man adult there is no more sight of the common epithelium by the completion of spliting. In the woman, on 
the contrary, the spliting is not completed, namely in the radix glandis is formed the common epithelium, as ever. The remaining of this special epithelium would be enough to remind the existence of the intraepithelial nerve fibres like in the embryo.

4. Melanin-pigments in the papillæ are seen in both plates of præputium remarkably, while in the glans clitoridis never. Among the epitheliums the tallest is seen in the inner plate of præputium, the next in the glans and the lowest in the outer plate of præputium. The papillæ are formed strongly, above all in the glans and the inner plate of præputium. This powerful formation of papillæ indorses generally by many of the works from our laboratory, that the clitoris is also rich in the sensory nerve fibres especially in its papillæ.

5. The nature of the epitheliums in clitoris. The epitheliums of both plates of præputium has str. corneum followed by str. granulosum and is characterized with the ceaseless cornification, like in the general skin with hairs. The epithelium of glans clitoridis, on the contrary has neither str. corneum nor str. granulosum and is covered only with a horny plate which probably thrusts off periodically as in the palatum durum or in the medium zone of anus. This epithelium should, therefore, belong to the mucous epithelium, accordingly the subepithelial connective tissue to the lamina propria mucosæ, just like in the glans penis.

The Outlines of the Sensory Innervation of Clitoris in Adult.

Through the observation of the silver preparations of clitoris in adult the most amazing for the author is that the sensory nerve elements reach to the exceeding development, compared with those in 10th month embryo. Namely, in the embryo, it is only Pacinian corpuscles which are found as corpusclar terminations, and the other sensory terminations are formed as unbranched or simply branched terminations extending scatteringly in the glans and the præputium. In the adult, on the contrary, the sensory nerve fibres distribute almost closely in the order of the glans, the inner and the outer plate of praputium, and the development of the terminations are also remarkable. To say nothing of the complicated branched terminations, there are found as the corpusclar terminations the various kinds of so-called genital nerve corpuscles besides the remarkably developed Pacinian corpuscles. To my surprise moreover, these sensory terminations exist far closely than those of penis in adult, compared with the preparations of penis conserved in our laboratory. This should be based upon that the sensory fibres are contained in clitoris also probably in the same proportion as in penis.

It must be added, however, that there are such sensory terminations as having already degenerated and disappeared too. It means that 
the special intraepithelial nerve fibres seen in the common epithelium to the glans and the præputium fall with the proceeding of the spliting of the epithelium since the embryonic end-period gradually in degeneration and desappearance. That is, in the epithelium of the completed glans and the præputium is seen no more entrance of the sensory fibres.

\section{Pacinian Corpuscles.}

Their localization and quantitative relation in clitoris are already cleared in 10th month embryo. It can be same in the adult also. In the Pacinian bodies formed in the surroundings of corpora cavernosa and along the dorsal nerves their long axis agrees generally with the course of the nerves, while in those formed in or rarely inside the tunica albuginea of the corpora their axis shows no special relation to the course of the nerves. Furthermore, in the corpuscles found beneath the corpora their axis crosses sometimes the axis of the corpora. Pacinian bodies exist else in the præputium and rarely in the septula of the corpora too (Fig. 1).

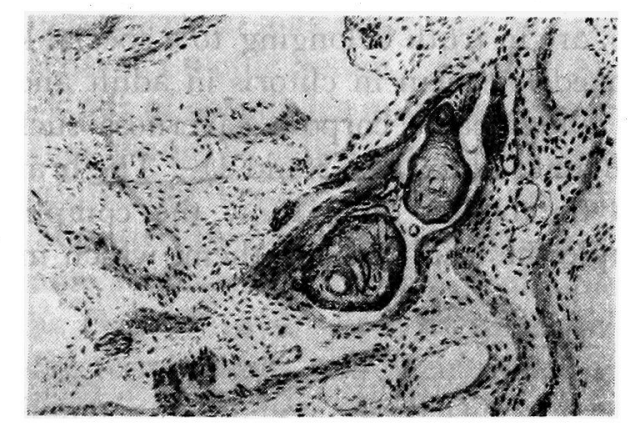

Fig. 1. Pacinian corpuscles seen in the septula of corpus cavernosum clitoridis in adult. Seto's impregnation, $\times 80$, reduced to $1 / 2$.

Pacinian corpuscles in the adult clitoris develop generally in the same grade: It happens rarely, to show the different development gradations based upon their localization, as in the embryo, though there are among them more or less differences in their sizes. The number of the lamellæ is about 20, their nuclei are clearly flat, and the inner bulb is formed relatively small, compared with that in the embryo, and filled with the fine granular, darkly red stained protoplasm including many specific nuclei. The nerve elements in it seem to develop remarkably after the birth and are mostly composed of 2-7 branches. These end-branches are in general separated in 2 kinds of thin and thick fibres, run forwards with their special winding nearly parallel to the long axis of the inner bulb and show frequently crossing or looping (Fig. 2). The thick fibres often show 


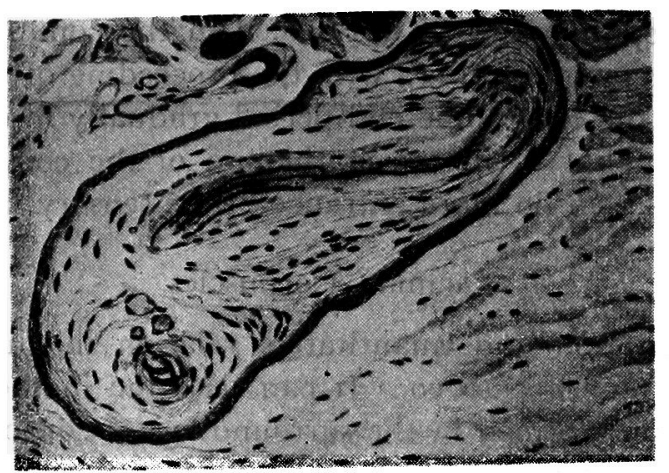

Fig. 2. Pacinian corpuscle formed along $\mathbf{n}$. dorsalis clitoridis in adult. Same staining, $\times 320$, reduced to $1 / 2$.

in their course strong fibrillar distributions. The end fibres terminate sharp or blunt, sometimes with small knobs.

In order to determine whether the sensory terminal bodies, which are recently found by me in clitoris in embryo and characteristic with the welling inner bulb, are in truth belonging to a special kind of the terminal bodies, I have looked for them in clitoris in adult and been able to find inside the tunica albuginea of corpora cavernosa such bodies as having the relatively wide inner bulb (Fig. 3 and 4). The branches of the sensory fibres entering to the bodies often show the complicated arrangement (Fig. 3), for example the thin fibres of them, which make here and there

Fig. 3

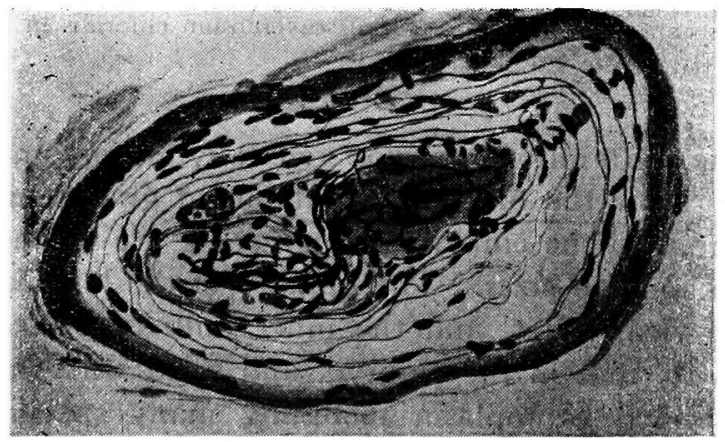

Fig. 4

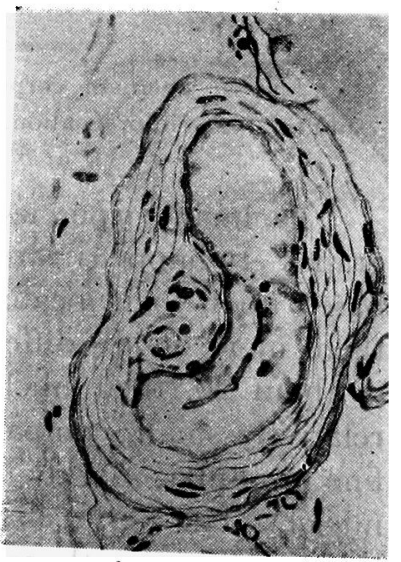

Fig. 3. Pacinian corpuscle with a wide inner bulb formed in tunica albuginea of corpus cavernosum clitoridis in adult. Same staining, $\times 320$, reduced to $1 / 2$. Fig. 4. Pacinian corpuscle with a wide inner bulb formed along $\mathrm{n}$. dorsalis clitoridis in adult. Same estaining, $\times 500$, reduced to $1 / 2$. 
small knobs, wind irregularly round the thick fibres in various modes. But as the bodies are in possession of 5-10 layers of lamellx in the surroundings of the inner bulb, they should be also clearly a kind of Pacinian corpuscles. It means that in clitoris of embryo are seen such Pacinian bodies as having very poorly developing lamellæ.

\section{Genital Nerve Corpuscles.}

The investigations of these bodies by the silver methods were carried out by Dahl, ${ }^{1)}$ Geller, ${ }^{8)}$ Mabuchi, ${ }^{51}$ Ohmori, ${ }^{71}$ Tello, ${ }^{10}$ ) Matsuda ${ }^{6)}$ and Ikui.4) Above all, Ikui has described in detail on the bodies in labium minus pudendi especially on the essence of their so-called inner bulb.

The genital nerve corpuscles, which were not able to be traced within clitoris in 10th month embryo yet, are formed in adult richly and completely and composed of various formations. It is thought, therefore, that they will be .gradually formed after the birth and completed near the puberty.

Now, as the corpusclar terminations except Pacinian corpuscles in the external genitals have been so long cited Krause's end-bulbs, Krause's genital bodies and Meissner's tactile bodies. Dogiel' ${ }^{2}$ found by methylenblue staining these 3 kinds of corpuscles in glans penis and its præputium and maintained that they should be called together genital nerve corpuscles, because no essential difference among them was recognized. His views generally have been admitted by many authorities.

Between the Krause's end-bulbs and the genital bodies there is no essential difference, as Dogiel ${ }^{2}$ says. Both of them belong to the same kind of the glomerular terminations and their difference consists in the size of their scale. The tactile bodies, which Dogiel recognizes in clitoris too, can not be found by me not only in clitoris, but also in the external genitals of the man. By the way, the morphological difference among these 3 kinds of corpuscles consist in the various distributions of the sensory fibres entering to the inner bulb and in the various arrangements of the special nuclei in it. It is, therefore, a great problem whether the special nuclei appear or not in the staining. Namely, the right morphological distinction is drawn first only by the staining. The tactile boedis are morphologically more or less different from both of the formers (Wada) ${ }^{12}$ ) found mainly in the papillæ of the palm and the sole and have been taken so long as the terminations concerning the tactile sense.

Our silver method stains the special nuclei in the inner bulb more clearly than the general staining method and the sensory elements, which

- enter the inner bulb, to make each specific termination, also so beautifully that the histological pictures of these sensory terminations are quite splendid. 
Now the corpusclar terminations formed in clitoris except the Pacinian bodies are by the detailed observation divided into 3 types. This views agree with those in penis. By the way, as these bodies are seen mainly in the external genitals and seem to have more or less morphological and physiological similarities, I should like to call these 3 kinds of corpuscles collectively the genital nerve bodies.

On the terminations of the type 1 and 2 Ikui $^{4}$ described recently in labium minus pudendi. Having the opportunity to observe his preparations and to compare them with my preparations of clitoris, I have known, that these both types are in clitoris far superior not only in mass, but also in scale to those in labium minus. Those of the type 3 are described by me first, as I know, and formed mainly in the strong connective tissue.

The inner bulb is in many authoritative text-books fine granular or gelatinous substance (Retzius, Ruffini) having no nuclei in general. Besides, it consists of connective tissue or bright substance. On the contrary, only Petersen ${ }^{8)}$ says, in agreement with Boeke's opinion, that Krause's end bulb is surrounded by a weak connective capsule and includes many of cells, which relate closely to the nerve elements entering the corpuscle, belong probably to the peripheral glia cells as those in the tactile body, but show the irregular arrangement unlike the latters.

Ikui") uses for the inner bulb the term of "end-nerve sheath" from the following reason. His views are clearly confirmed by my observation in clitoris too, that is the inner bulb is composed of the bright, but fine granular ground substance and in the type 1 many of, in the type 2 a few of flattered special nuclei in it and show a synzytial nature having no cell border. This common protoplasm is evident especially in the type 2 and often represented as the continuation of the thick sheath covering the nerve stem which passes into it. This views are cleared also within the Krause's end bulb (Fig. 252) in the Stöhr's Histology') (1940), though the special nuclei are not shown. What is this thick special sheath made of? This is by the mylin-staining unstainable, it might, therefore, belong to the Schwann's sheath like the opinion of Petersen.8) Schwann's sheath, whose existence is supposed generally only by its nuclei, because its protoplasm is scarcely seen. But it becomes often a thick nerve sheath, as the nerve fibre approaches to its termination, and passes into the termination, to form finally the essence of the inner bulb.

It is lately considered that the special cells in the various nerve terminations would be either physiologically or histologically of endocrine. The inner bulb of the genital nerve bodies in clitoris is also thought of the same nature. It produces probably through the stimulus from the outside some endocrinous chemical substances which irritate secondarily the terminal nerve elements in this part. 
Describing about the genital nerve bodies of 3 types, the terminations of the type 1 (Fig. 5) exist mostly in propria of glans clitoridis, followed by the inner and the outer plate of præputium, and is often found in the corpora cavernosa too. It is for this type characteristic that the nerve elements entering the inner bulb branch and anastomose repeatedly, to show as a whole a typical, complicated glomerular formation wide expanded in the territorium. This type is, like the type 2, divided into the capsulated from having a distinct connective capsule and the non-capsulated without it.

The nerve elements forming this glomerulum are composed of many branches showing scarcely the change of thickness, run with the complicated course, anastomose each other and show often the arrangement like a tangle of threads. The special nuclei in the inner bulb are oval or round, arrange very irregularly and often exist great in number.

The terminal bodies of the type 2 is the most in the propria of glans clitoridis, then in the following order of the inner plate, the cavernous body of glans especially its surroundings, the outer plate, the periphery of corpora cavernosa and the tunica albuginea. The characteristics of the type 2 consist in, as Ikui ${ }^{4}$ describes, that only the center part of the inner bulb is occupied by the distribution of the terminal fibres, while its periphery is widely filled with the substance of the inner bulb (Fig. 6). But it happens sometimes the nerve elements extend nearly in the whole ter-

Fig. 5

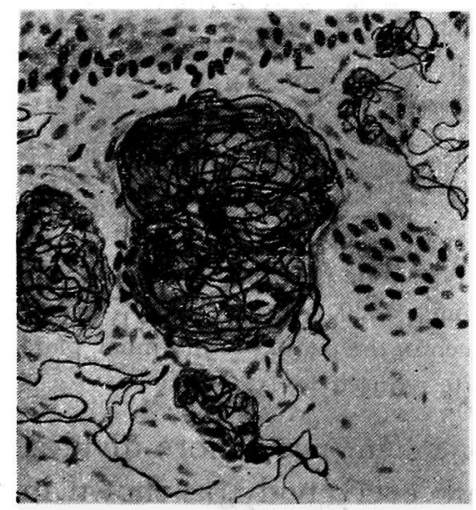

Fig. 6

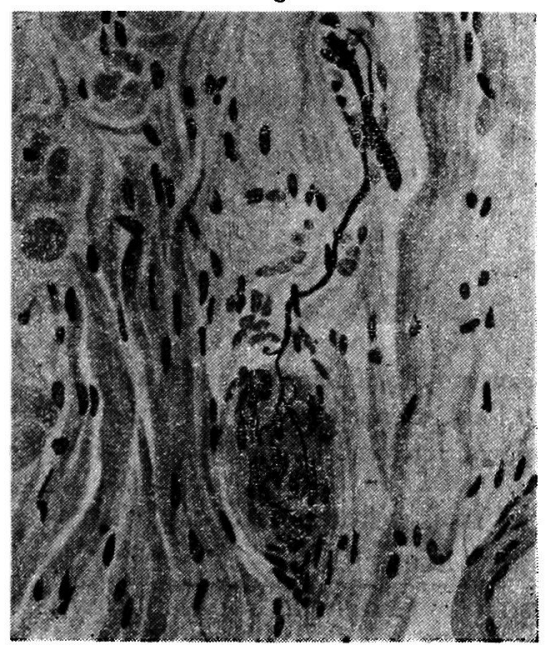

Fig. 5. Genital nerve corpuscles of type 1 showing the typical glomerular termination found in tunica propria of glans clitoridis in adult. Same staining, $\times 500$, reduced to $1 / 2$. Fig. 6. Isolated genital nerve corpuscle of type 2 in the surroundings of corpus cavernosum clitoridis in adult. Same staining, $\times 320$, reduced to $2 / 3$. 
ritorium (Figs. 7 and 8 ). Now the differentiation to the type 1 consists in the nature of the nerve elements as well as their arrangement. Namely in the type 2 they are represented generally by the thick fibres showing the change of thickness. Their branching is relative few and the end branches take the special winding course, to terminate finally with fibrillar distributions or button-formed swellings, but often blunt or sharp. . There are often such terminations too, that by the low magnification they seem to be genuine glomerula, but by the high magnification especially with the oilimmersion they make no anastomosis between the branches. The arrangement of the special nuclei is very irregular like in the type 1.

The terminations of the type 2 is further divided into the solitary and the aggregated form. It is for the latter characteristic, that 6-8 of corpuscles gather together and are enclosed by a common sheath (Fig. 7). The terminations of the type 2 are formed generally round or oval, but often long and narrow (Fig. 8).

Fig. 7

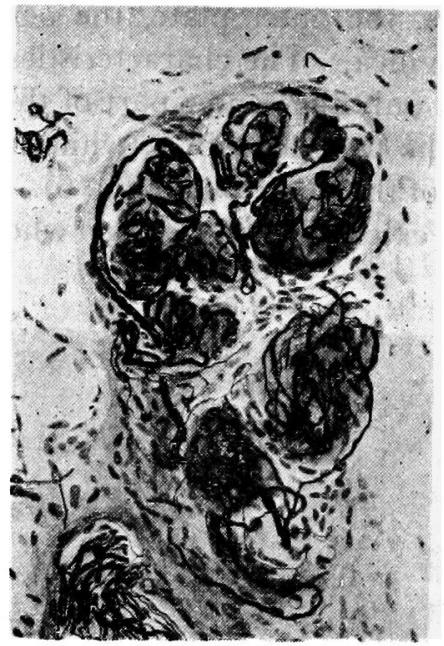

Fig. 8

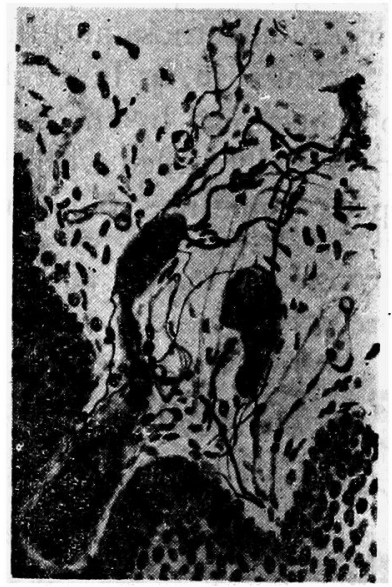

Fig. 7. Aggregated genital nerve corpuscle of type 2 in the surroundings of corpus cavernosum of glans clitoridis in adult. Same staining, $\times 320$, reduced to $1 / 2$. Fig. 8 . 3 long extended genital nerve corpuscles of type 2 seen in the papilla of the inner plate of præputium clitoridis in adult. Same staining, $\times 320$, reduced to $1 / 2$.

It is common to all the types, that one stem nerve fibre divides into many branches $(2-5)$, which pass over into every body, as shown in the figures. This speciality happens especially on the sensory fibres distributing in the external genitals.

The terminations of the type 3 are found relative richly in such special places of clitoris as consisting of strong connective tissue, i.e. in tunica albuginea of corpora cavernosa especially between or often in its both 
layers, in the septulæ of corpus cavernosus glandis, besides beneath the glans body.

These bodies which show every special formation might prefer to be called complicated branched terminations. Because the special nuclei exist simply here and there along the nerve fibres, so that there is no formation of the inner bulb, accordingly no existence of the connective capsule too. As their physiological significance, however, is thought to be similar with that of the both former terminations, and the termination style of the nerve elements resembles also to that in the type 2, so I should like to call this special terminations the genital nerve corpuscles of the type 3 . As shown by Figs. 9, 10 and 11, a thick medullated fibre entering the strong connective tissue (Fig. 9) loses its myelin just before the terminal

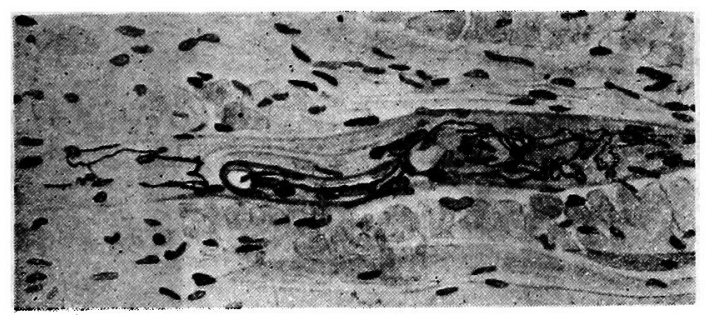

Fig. 9. Genital nerve termination of type 3 found between the both layers of tunica albuginea of corpus cavernosum clitoridis in adult. $m$ myelin. Same staining, $\times 320$, reduced to $2 / 3$.

formation and divides into thick branches showing the change of thickness, which take every special winding course without anastomosing each other, to terminate sharp or blunt, or often with various end formations and to be finally as a whole represented as somewhat long extended ter- minations showing irregular arborescent or sometimes glomerular arrangement.

Now these terminations are seen in the tunica albuginea of penis too, as the preparations conserved in our laboratory show evidently. They

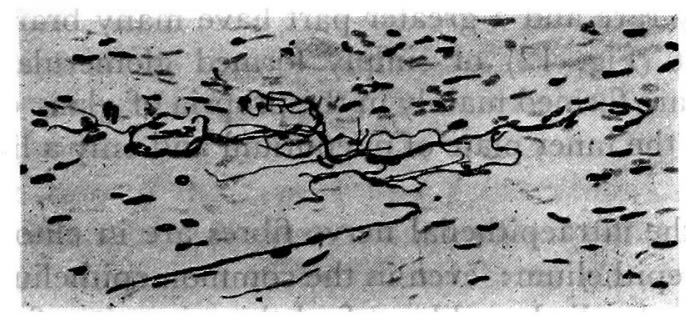

Fig. 10. Ditto. The nerve elements show a arborescent distribution without connective capsule. Same staining, $\times 320$, reduced to $2 / 3$. 
are greater than those in clitoris in size. But the density of their distribution is in the latter far superior, though it is considered probably that there is no difference between both of them in the total of the terminations.

Fig. 11

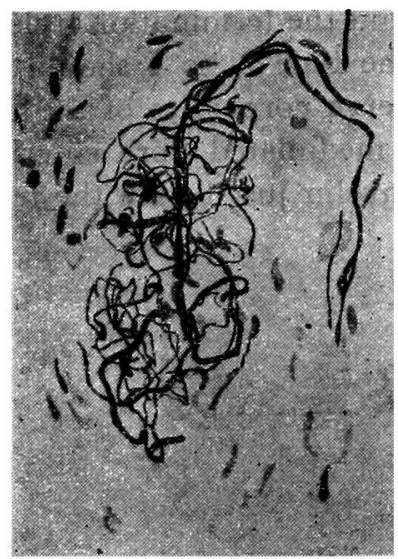

Fig. 12

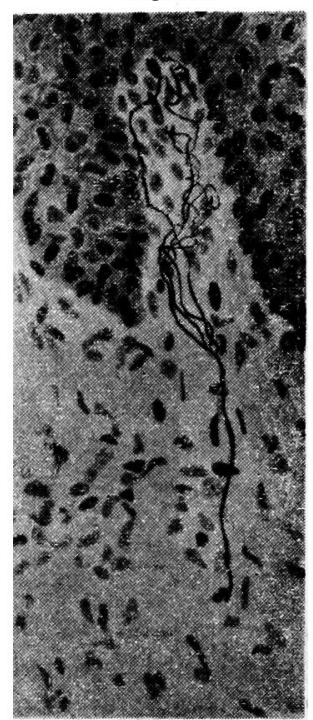

Fig. 11. Genital nerve corpuscle of type 3 showing a glomerular distribution of nerve elements found beneath glans clitoridis in adult. Same staining, $\times 500$, reduced to $1 / 2$. Fig. 12. Simply ramified, arborescent termination formed in a papilla of the inner plate of præputium clitoridis in adult. Same staining, $\times 320$, reduced to $2 / 3$.

\section{Ramified Terminations.}

The sensory terminations in clitoris are in the embryo, except the Pacinian corpuscles, represented by the unbranched or simply branched terminations, in the adult on the contrary mostly by the corpusclar terminations and partly by the ramified, and the non-ramified are scarcely seen. Of the branched terminations, those simply consisting of 2-3 branches are very rare and a greater part have many branches and show every arborescent (Fig. 12) or simply formed glomerular arrangement (Fig. 13). They are formed mainly in the propria of glans especially in its papillæ, partly in the inner plate of præputium and only a little in its outer plate too.

At the end, the intraepithelial nerve fibres are in clitoris in adult no more seen in any epithelium: even in the common epithelium remained in the radix corporis glandis I could not find them in spite of my expectation for their existence. 


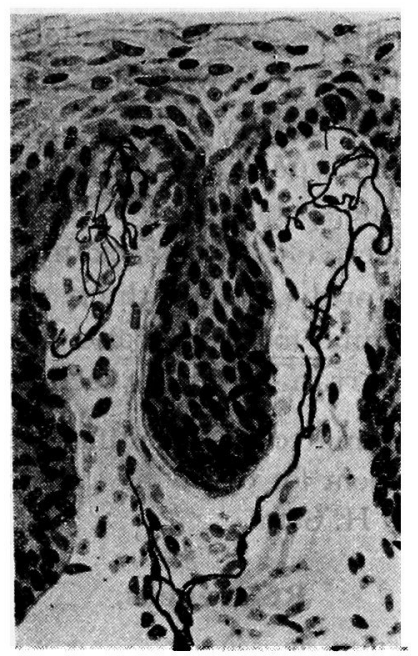

Fig. 13. Simply ramified, glomerular termination formed in the papillæ of the outer plate of præputium clitoridis in adult. Same staining, $\times 320$, reduced to $2 / 3$.

\section{Summary.}

The sensory terminations in clitoris in adult show remarkable development, compared with those in the embryo of 10th month, but with a exception of the intraepithelial fibres which exist no more even in the common epithelium remained in the radix clitoridis.

Such a special corpusclar termination as having a swelling inner bulb in the embryo seems to belong to a Pacinian corpuscle, because it show in adult in its periphery the formation of some of lamellæ.

The corpusclar terminations except the Pacinian corpuscles in the external genitals in adult have long included Krause's end bulbs, Krause's genital bodies and Meissner's tactile bodies. But there is no essential difference between both of the formers. And the existence of the latters is denied too. The morphological difference of these bodies consists in the conditions of the arrangement of the sensory nerve fibres and special nuclei in the inner bulb.

These terminal bodies are divided into 3 types. But, as they have morphologically more or less similarities, they should be called collectively the genital nerve bodies.

Each termination of the type 1 and 2 has remarkable inner bulb, which is showed as a synchytium consisting of fine granular substance and special nuclei and considered to be of peripheral glia and of endocrine nature.

The terminal bodies of the type 1 are characteristic with the glomerular 
arrangement of the nerve fibres extending widely in the whole territorium of the inner bulb, while the type 2 with the nerve distribution mainly in the center part of the inner bulb.

The terminations of the type 3 , which have no formation of the inner bulb and could be called the special ramified terminations too, are seen in general in the strong connective tissue, for example in the tunica albuginea of corpora cavernosa clitoridis. The thick medullated fibre loses its myelin just before the terminal formation and divides into many branches, which terminate with the arrangement as seen in the type 2.

Through the Grant Committee for Scientific Researches a grant was given from the Education Department for defraying the expenses of this work, which is gratefully acknowledged. $\mathbf{H}$. Seto.

\section{References.}

1) Dahl, Z. f. Geburtsh., 1916, 78, 539.

2) Dogiel, Arch. mikr. Anat., 1893, 41, 585.

3) Geller, Z. . Gyn., 1922, 47, 623.

4) Ikui, Tohoku Igaku Zassi, 1949, 39, 104.

5) Mabuchi, Mitt. med. Facul. Univ. Tokyo, 1924, 31, 385.

6) Matsuda, Kaibogaku Zassi, 1935, 8, 532.

7) Ohmori, Z. f. Anat., 1924, 70, 347.

8) Petersen, Histologie u. mikr. Anat., VI, p. 852, 1935, J. F. Bergmanna München.

9) Stöhr, Lehrbuch der Histologie (Möllendorff), 24. Auflage, 1940.

10) Tello, Trav. lab. rech. biol. Madrid, 1932, 28, 1.

11) Yamada, Tohoku J. Exp. Med., in press, this volume p. 151.

12) Wada, Tohoku Igaku Zassi, 1949, 39, 73. 